\title{
TENSOR-BASED QUALITY PREDICTION FOR BUILDING MODEL RECONSTRUCTION FROM LIDAR DATA AND TOPOGRAPHIC MAP
}

\author{
B. C. $\operatorname{Lin}^{1 *}$, R. J. You ${ }^{2}$ \\ Department of Geomatics, National Cheng Kung University, 1 University Road, Tainan City, Taiwan - \\ ${ }^{1}$ cclin0520@gmail.com \\ ${ }^{2}$ rjyou@mail.ncku.edu.tw
}

KEY WORDS: LiDAR, tensor analysis, robust least squares, data fusion, building model reconstruction

\begin{abstract}
:
A quality prediction method is proposed to evaluate the quality of the automatic reconstruction of building models. In this study, LiDAR data and topographic maps are integrated for building model reconstruction. Hence, data registration is a critical step for data fusion. To improve the efficiency of the data fusion, a robust least squares method is applied to register boundary points extracted from LiDAR data and building outlines obtained from topographic maps. After registration, a quality indicator based on the tensor analysis of residuals is derived in order to evaluate the correctness of the automatic building model reconstruction. Finally, an actual dataset demonstrates the quality of the predictions for automatic model reconstruction. The results show that our method can achieve reliable results and save both time and expense on model reconstruction.
\end{abstract}

\section{INTRODUCTION}

The airborne LiDAR technique has been extensively adopted for the purpose of quickly acquiring a large number of highly qualitative point clouds, and it has become widely implemented in $3 \mathrm{D}$ building models. LiDAR data provides an accurate representation of building surfaces, but since it has poor texture information, accurate building boundary extraction from LiDAR data may be difficult to obtain (Maas and Vosselman 1999). Therefore, the building boundaries can be implemented by using additional data sources, such as $2 \mathrm{D}$ topographic maps.

Since a data fusion of LiDAR data and 2D map information takes advantage of both surface and boundary information, a great many researchers have investigated it in order to reconstruct buildings (Maas and Vosselman 1999; Vosselman and Dijkman 2001; Filin 2002; Overby et al. 2004). In general, building roof patch features are first extracted from LiDAR data Next, building models are reconstructed by combining the building boundaries obtained from ground plans and the intersection lines of adjacent planar faces derived from LiDAR data.

However, the coordinate systems of LiDAR data and 2D maps are often different. To overcome the problem of coordinate systems of various data sources, data registration is a critical step for fusion of LiDAR data and the topographic map information (Schenk and Csatho 2002; Filin et al. 2005; Gruen and Akca 2005; Park et al. 2006).

In this study, plane segments in LiDAR data are extracted in the feature space based on the tensor voting computational framework (Medioni et al. 2000). The tensor voting algorithm implements features such as faces, lines and points through a symmetric tensor field directly derived from data. All geometric structures (surfaces, lines and points) can therefore be inferred simultaneously.
For data registration, the transformation parameters between LiDAR data and topographic maps are estimated using a robust least squares method (RLS). After registration, height information derived from LIDAR data is involved in topographic maps and then the spatial positions of building outlines can be reconstructed. To completely reconstruct a 3D building model, the roof ridges extracted from LiDAR data should be added (You and Lin 2011a).

To achieve reliable results, a quality indicator derived from tensor analysis based on the residuals of the boundary point is introduced. The indicator can be use for checking the correctness of the building model in an automatic reconstruction process. Therefore, both time and expense on model reconstruction can be saved.

In the following, the feature extraction based on the tensor voting method is first described. In section 3, the data registration and residual tensor analysis are address. Finally, the residual tensor on different building cases of actual airborne LiDAR dataset is analyzed.

\section{FEATURE EXTRACTION BASED ON TENSOR VOTING METHOD}

In this study, the tensor voting method (TVM) is adopted to extract roof faces from LiDAR data since this method can sufficiently consider the geometric relationships between surrounding points. In the TVM, a second order tensor field should be first constituted, and then planar features, namely roof faces, can be extracted from irregularly distributed LiDAR point clouds (You and Lin 2011a).

The geometric feature of a point can be described by a secondorder symmetric tensor which is expressed as follows:

\footnotetext{
* Corresponding author.
} 


$$
\mathbf{T}=\left[\begin{array}{lll}
\mathbf{e}_{1} & \mathbf{e}_{2} & \mathbf{e}_{3}
\end{array}\right]\left[\begin{array}{ccc}
\lambda_{1} & 0 & 0 \\
0 & \lambda_{2} & 0 \\
0 & 0 & \lambda_{3}
\end{array}\right]\left[\begin{array}{l}
\mathbf{e}_{1} \\
\mathbf{e}_{2} \\
\mathbf{e}_{3}
\end{array}\right],
$$

where $\mathbf{e}_{1}, \mathbf{e}_{2}$ and $\mathbf{e}_{3}$ indicate three independent and orthogonal eigenvectors; $\lambda_{1}, \lambda_{2}$, and $\lambda_{3}$ are eigenvalues with respect to the eigenvectors $\mathbf{e}_{1}, \mathbf{e}_{2}$ and $\mathbf{e}_{3}$. The eigenvalues are real and $\lambda_{1} \geq \lambda_{2} \geq \lambda_{3}$ if $\mathbf{T}$ is a positive-semidefinite tensor.

The tensor voting method is used for deriving the implied vector information in LiDAR point clouds. The kernel of the tensor voting is the tensor communication among points. Each point receives vector information from its surrounding points and stores the vector information by the tensor addition rule. The resultant tensor can be expressed as follows:

$$
\mathbf{T}=\sum_{i=1}^{m} w_{i} \mathbf{T}_{i}^{p}
$$

where $w$ is a Gaussian decay function depending on the Euclidean distance between the receiving site and the voting site.

After the tensor communication is completed, the geometric feature information, such as planar, linear and corner features, can now be captured according to the rules of geometric features mentioned in Medioni et al.(2000). However, the points in the border region receive fewer votes than the points in the central region do, so that eigenvalues $\lambda_{1}, \lambda_{2}$, and $\lambda_{3}$ are generally smaller in the border region of an object than in the central region of the same object. To reduce the effect of the number of points, the planar feature indicators $\lambda_{1}-\lambda_{2}$ may be normalized as

$$
\mathrm{c}=\frac{\left(\lambda_{1}-\lambda_{2}\right)}{\lambda_{1}}
$$

The normalized value of planar strength is introduced for the planar feature extraction and the region growing in this study, since it is the sensitive indicator for planar features (You and Lin 2011b).

The region-growing method is adopted for the segmentation of the points with similar planar features. The region-growing method used here is based on the homogeneity of the principal features. The principal features are the normalized planar feature strength $\mathrm{c}$ and the corresponding normal vectors in this study. In region-growing, only the points with a normalized planar feature strength $\mathrm{c}$ greater than a threshold can be adopted as seed points. The threshold is recommended to be 0.96 or larger in our experiments.

First, the point that has the largest c-value is chosen as the seed point for the planar feature extraction. A point is merged into the region if both the c-value and the directional difference of the normal vector of that point are less than the corresponding thresholds. Then, the point with the second largest c-value in the LiDAR data, excluding all extracted points in the segment associated with the first seed point, is adopted as the second seed point for growing the next segment. This region-growing procedure proceeds until no more seed points are available. Figure 1 illustrates segmentation result after region growing.

In this study, ridge lines are inferred by the intersection of two adjacent roof faces segmented from LiDAR data, as recommended by Maas and Vosselman (1999). According to the rule that the triangles on the outer boundary of a triangular irregular network (TIN) mesh have only one or two neighboring triangles (Pu and Vosselman 2007), a TIN structure is adopted to extract boundary points.

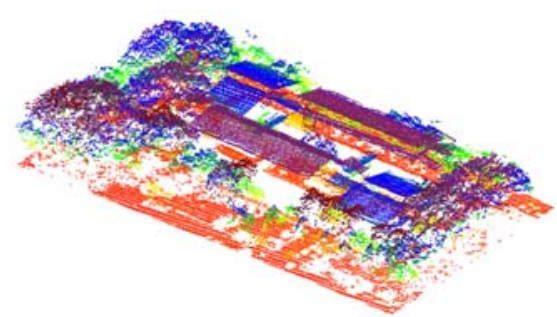

(a)

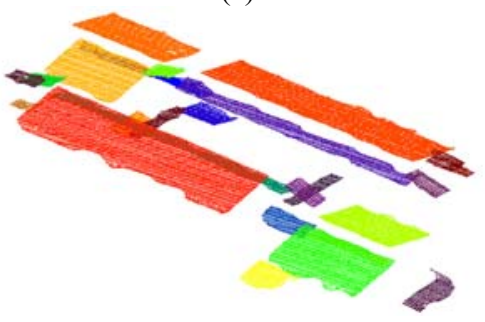

(b)

Figure 1. (a)LiDAR points and (b)the segmentation result.

\section{DATA REGISTRATION AND TENSOR ANALYSIS OF RESIDUALS}

\subsection{Data registration}

In this study, LiDAR data and topographic maps are integrated for building model reconstruction. Hence, data registration is intended to transform these two datasets into a common coordinate system. The discrepancies between boundary points and building outlines before data registration are shown in Figure 2. To determine the transformation parameters, the robust least squares (RLS) matching of boundary points and building outlines on a local xy-plane are used (You and Lin 2011a).

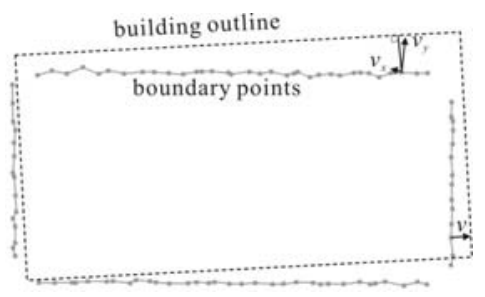

Figure 2. Boundary points and ridge lines. 
In order to register the boundary LiDAR points of buildings to the corresponding outline segments, a $2 \mathrm{D}$ similarity transformation is adopted:

$$
\left[\begin{array}{l}
x^{\prime} \\
y^{\prime}
\end{array}\right]=\left[\begin{array}{cc}
w & u \\
-u & w
\end{array}\right]\left[\begin{array}{l}
x \\
y
\end{array}\right]+\left[\begin{array}{l}
r \\
s
\end{array}\right],
$$

where $(\mathrm{x}, \mathrm{y})$ are the horizontal coordinates of a LiDAR point in a local coordinate system; $\left(x^{\prime}, y^{\prime}\right)$ are the new coordinates in the map system after the transformation and $(r, s)$ are the shifts of the origin. $\mathrm{w}=\mathrm{m} \cos \alpha$ and $\mathrm{u}=\mathrm{m} \sin \alpha$, where $\alpha$ is the rotation angle, and $\mathrm{m}$ is the scale factor.

Assume that boundary LiDAR points with the new coordinates should fall exactly on an outline segment $L$ : $a x^{\prime}+b y^{\prime}+c=0$. Substitution of Eq. (5), followed by introducing measurement errors in the coordinates of the boundary points, leads to:

$$
\begin{aligned}
& \left(a\left(x+v_{x}\right)+b\left(y+v_{y}\right)\right) w+\left(-b\left(x+v_{x}\right)+a\left(y+v_{y}\right)\right) u \\
& +a r+b s+c=0
\end{aligned}
$$

where $(a, b, c)$ can be calculated from the corresponding polygon data of an outline segment, and the residuals $v_{x}$ and $v_{y}$ represent two components of the distance vector $\mathrm{v}$ from a LiDAR point to the corresponding outline segment.

The registration process is performed using the iterative RLS method (Klein and Foerstner 1984). The objective function in RLS consists of the sum of squares of the distances from boundary points to building outlines on a local xy-plane. In each iteration of the RLS adjustment, the corresponding outline segment for each boundary LiDAR point located now by new transformed coordinates must be re-determined. The procedure proceeds until the estimated standard deviation of the distances is convergent.

\subsection{Tensor analysis of residuals}

A resultant tensor $\mathbf{T}_{\mathrm{r}}$ is used to analyze the registration result of each building (You and Lin 2011a).

$$
\mathbf{T}_{r}=\sum_{i} \mathbf{T}_{i}=\sum_{i=1} \mathbf{v}_{i} \mathbf{v}_{i}^{\mathrm{T}}
$$

where the residual tensor $T_{i}$ for each boundary point is obtained from the estimated residual vector $\hat{\mathbf{v}}_{i}=\left[\hat{v}_{x_{i}} \hat{v}_{y_{i}}\right]^{\mathrm{T}}$, and then the residual tensors of all boundary points of a building are added together to form a resultant tensor $\mathbf{T}_{\mathrm{r}}$. To reduce the influence of the number of the points, the resultant tensor is normalized by dividing the number of the boundary points in this study.

Based on the fact that the boundary points of a building surround the closed building polygon, it is evident that the resultant tensor $\mathbf{T}_{\mathrm{r}}$ is positive semi-definite and a rank-2 tensor. The eigenvalues $\left(\lambda_{\max }, \lambda_{\min }\right)$ and eigenvectors $\left(\mathbf{e}_{\max }, \mathbf{e}_{\min }\right)$ of the normalized resultant tensor can be derived by tensor decomposition as follows:

$$
\mathbf{T}_{\mathrm{r}}=\left[\begin{array}{ll}
\mathbf{e}_{\max } & \mathbf{e}_{\min }
\end{array}\left[\begin{array}{cc}
\lambda_{\max } & 0 \\
0 & \lambda_{\min }
\end{array}\right]\left[\begin{array}{l}
\mathbf{e}_{\max }^{\mathrm{T}} \\
\mathbf{e}_{\min }^{\mathrm{T}}
\end{array}\right],\right.
$$

where $\lambda_{\max } \geq \lambda_{\min } \geq 0$. This tensor can be geometrically visualized as an ellipse [22]. The eigenvectors represent the orientation of the ellipse, and the root square values of the eigenvalues represent the lengths of the principal axes.

After registration, the tensor ellipse of each building polygon can be determined. If the tensor ellipse is beyond the tolerance circle, it means that the boundary LiDAR points are not sufficient to match the building outlines. This implies that these discrepancies may influence the results of the reconstruction. Therefore, it is possible to identify which building models are not reconstructed well using residual tensor analysis.

\subsection{Building model reconstruction}

After registration, an automatic reconstruction of $3 \mathrm{D}$ building models is applied. In this procedure, the height of each building outline node can be determined by the plane equation of a LiDAR surface segment, and then the structural lines derived from LiDAR data and the building outlines are automatically connected according to following rules (Lin et al. 2010):

1. The 3D structural lines projected on the local xyplane should be first extended to the boundary lines when they are shorter than they should be.

2. If the intersection point of a structural line and a boundary line is near a node point within a small region, the structural line is directly connected to the node point (case A in Figure 3).

3. If the height of a structural line at the intersection point is not different from the height of the boundary line, the structural line is directly connected to the boundary line and a new node of the boundary line is added (case B in Figure 3).

4. If the height of a structural line at the intersection point is significantly different from the height of the boundary line, two new additional structural lines may be needed(case C in Figure 3).

Then a $3 \mathrm{D}$ building model can be reconstructed.

\section{EXPERIMENT AND ANALYSIS}

An airborne LiDAR dataset for a $350 \times 500 \mathrm{~m}^{2}$ experimental area was acquired by an Optech ALTM 30/70. The flying height for the laser scanning was $500 \mathrm{~m}$ AGL. The average LiDAR point density was $6 \mathrm{pts} / \mathrm{m}^{2}$. The horizontal and vertical precision was about $25 \mathrm{~cm}$ and $15 \mathrm{~cm}$, respectively. This dataset was referred to Taiwan geodetic datum 1997.0. The topographic map with a scale of 1:1000 for this area was produced from aerial images and is based on Taiwan geodetic datum 1967.

After registration, a resultant tensor can be determined to analyze the registration result. If the tensor ellipse is beyond the tolerance circle, it means the discrepancy between the boundary points and the outlines of that building is obvious. In an actual experimental dataset, we illustrate some cases as mentioned above and present how the resultant tensor analysis can identify the incorrect reconstruction model. These cases can be divided 
into two categories: (1) There are not enough boundary points from the LiDAR data to depict the complete building outlines, as shown in Cases I. (2) The building outlines are not detailed enough, as shown in Cases II. These two categories indicate that boundary LiDAR points do not sufficiently match the building outlines.

Case I: There are not enough boundary points from the LiDAR data to depict the complete building outlines.

(1) Building with a horizontal or slanted roof plane. In these buildings, the roof faces are partly or fully covered by neighboring building or trees.

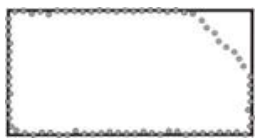

Figure 3. Building with a horizontal or slanted roof plane.

(2) Building with a ridge or hips. In these buildings, the important structural lines, like hips, are partly or fully covered by trees etc.

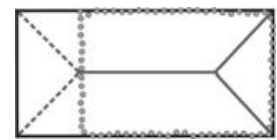

Figure 4. Building with a ridge or hips.

Case II: Building outlines are not detailed enough.

(1) Two adjoining buildings with flat or slanted roof planes or roofs with two different ridges. In these buildings, the building outlines of these two buildings are drawn together with one outline. The border line between these two buildings is not drawn in the topographic map.

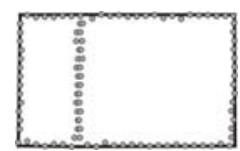

Figure 5. Two adjoining buildings with flat or slanted roof planes or roofs with two different ridges.

(2) Some small buildings on top of a big building. In this case, the outlines of the small buildings are not drawn on the topographic map.

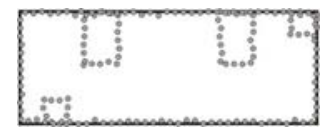

Figure 6 . Some small buildings on top of a big building.

According to these case analyses, the tensor ellipse is available for checking the incorrectly reconstructed models when the automatic reconstruction is completed. In our experience, it is good enough that the radius of the tolerance circle is set to 2 times the estimated standard deviation of the discrepancy.

After reconstruction, some of the building models may be incorrect when the discrepancy between the tensor ellipse and the tolerance circle at the buildings is significant. In Case I-1, a building with a single roof plane, the building models can be still reconstructed correctly, since the roof planes and the heights of the nodes can be determined by the LiDAR surface segments. In Case I-2, a building with multiple intersecting roof planes, the building model can be also reconstructed correctly as long as all of the structural lines of the building are extracted exactly or inferred correctly from the LiDAR data. The structural line can be still inferred correctly when a part of it is covered. However, if the structural line is completely covered, the reconstructed building model will be incorrect. A building with multiple non-intersecting roof planes, such as buildings with different heights of roof planes that are close to one another (Case II-1), or small structures on the roofs (Case II-2). The reconstructed building models are always wrong in these cases, since these small structures are usually not shown on the topographic map or the closely adjacent buildings are drawn with a single outline. All these incorrect cases can be observed in the tensor analysis stage.

Incorrect building models may occur in the results of the automatic reconstruction due to either insufficient LiDAR data or topographic map information. Hence manual refinement of the reconstructed building models may be necessary after the automatic procedure. Since the incorrectly reconstructed building models can be detected according to the tensor analysis developed in this study, it reduce both the time and cost of building reconstruction.

The quality of reconstructed building models is evaluated by tensor analysis. In Figure 5, a closed polygon represents a building. The results show that a total of 33 of 108 buildings are incorrect building models as shown with gray polygons in Figure 5.

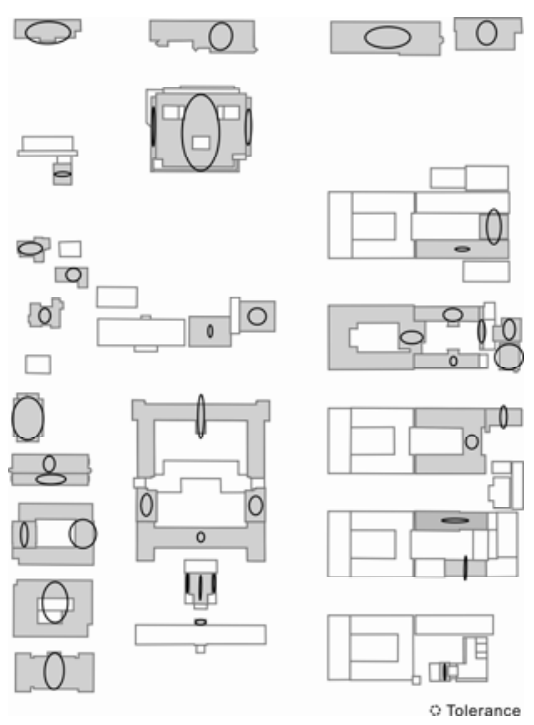

Figure 7. Incorrect building models (gray polygons) in the test area.

In Figure 7, a closed polygon represents a building. Our results have shown that in total, 41 tensor ellipses are beyond the tolerance circle. There are 6,5 and 30 buildings with single roof planes, multiple intersecting roof planes and multiple nonintersecting roof planes respectively. These building models should be manually checked after the automatic reconstruction procedure. The 6 buildings with single roof planes are automatically reconstructed very well, as mentioned before, and 
one of the five buildings with multiple intersecting roof planes is also reconstructed very well, since its ridge lines are completely inferred from Lidar data. The other 33 buildings cannot be reconstructed correctly since the important structural lines on their roofs are covered by trees or the outlines of small structures are not drawn on the topographic map. All of these incorrect models should be refined manually, by photogrammetry, outline estimation from Lidar data, and even field work, for instance.

\section{CONCLUSIONS}

The residual tensor ellipses presented here can be regarded as indicators to evaluate the quality of registration and to show where manual modification may be needed during model construction. The experiments have shown that the proposed method for the building reconstruction procedure with Lidar data and topographic map information, including feature extraction, registration, reconstruction and quality analysis, can be processed automatically and yields reliable results. Although manual editing is needed in order to achieve refined 3D building models, the results have shown that our method can save time and expense for model construction by using tensor ellipses.

\section{REFERENCES}

Filin, S. 2002. Surface clustering from airborne laser scanning data. International Archives of Photogrammetry and Remote Sensing, 34(3A), pp. 119-124.

Filin, S., Y. Kulakov and Y. Doytsher (2005). Application of Airborne Laser Technology to 3D Cadastre. FIG Working Week 2005 and GSDI-8, Cairo, Egypt.

Gruen, A. and D. Akca 2005. Least squares 3D surface and curve matching. ISPRS Journal of Photogrammetry and Remote Sensing, 59(3), pp. 151-174.

Klein, H. and W. Foerstner 1984. Realization of automatic error detection in the block adjustment program PAT-M43 using robust estimators. International Archives of Photogrammetry and Remote Sensing, 25(A3a), pp. 234-245.

Lin, B. C., R. J. You and M. C. Hsu (2010). Building Model Reconstruction With Lidar Data and Topographic Map by Registration of Building Outlines. ISPRS TC VII Symposium 100 Years ISPRS, Vienna, Austria, IAPRS.

Maas, H. G. and G. Vosselman 1999. Two algorithms for extracting building models from raw laser altimetry data. ISPRS Journal of Photogrammetry and Remote Sensing, 54(2-3), pp. 153-163.

Medioni, G., M. S. Lee and C. K. Tang (2000). A computational framework for segmentation and grouping, Elsevier Science, New York.

Overby, J., L. Bodum, E. Kjems and P. M. Ilsoe 2004. Automatic 3D building reconstruction from airborne laser scanning and cadastral data using Hough transform. International Archives of Photogrammetry and Remote Sensing, 35(B3), pp. 296-302.

Park, J., I. Lee, Y. Choi and Y. J. Lee 2006. Automatic Extraction of Large Complex Buildings Using Lidar Data and
Digital Maps. International Archives of Photogrammetry and Remote Sensing, 36(3), pp. 148-154.

$\mathrm{Pu}$, S. and G. Vosselman 2007. Extracting windows from terrestrial laser scanning. International Archives of Photogrammetry Remote Sensing and Spatial Information Sciences, 36(3/W52), pp. 320-325.

Schenk, T. and B. Csatho 2002. Fusion of LIDAR data and aerial imagery for a more complete surface description. International Archives of Photogrammetry and Remote Sensing, 34(3A), pp. 310-317.

Vosselman, G. and S. Dijkman 2001. 3D building model reconstruction from point clouds and ground plans. International Archives of Photogrammetry and Remote Sensing, 34(3/W4), pp. 37-43.

You, R. J. and B. C. Lin 2011a. A Quality Prediction Method for Building Model Reconstruction Using LiDAR Data and Topographic Maps. IEEE Transactions on Geoscience and Remote Sensing, 49(9), pp. 3471-3480.

You, R. J. and B. C. Lin 2011b. Building Feature Extraction from Airborne Lidar Data Based on Tensor Voting Algorithm. Photogrammetric Engineering \& Remote Sensing, 77(12), pp. 1221-1231. 